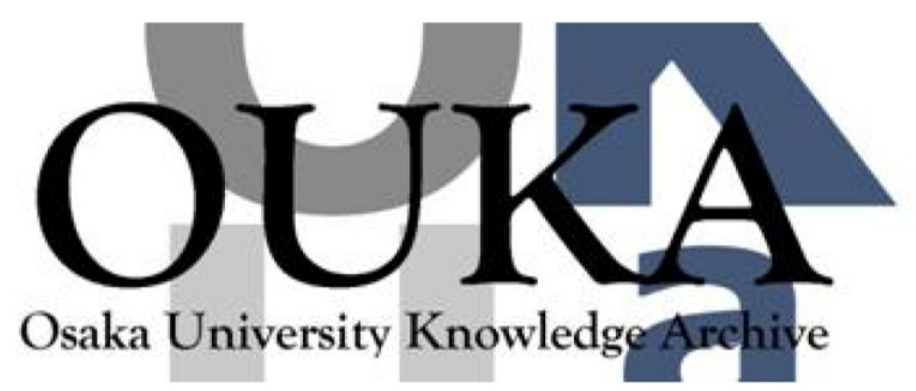

\begin{tabular}{|c|c|}
\hline Title & $\begin{array}{l}\text { Molecular dynamics analysis on wetting and } \\
\text { interfacial properties of water-alcohol mixture } \\
\text { droplets on a solid surface }\end{array}$ \\
\hline Author (s) & Surblys, D.; Yamaguchi, Y.; Kuroda, K. et al. \\
\hline Citation & $\begin{array}{l}\text { The Journal of Chemical Physics. 140(3) } \\
\text { p. } 034505\end{array}$ \\
\hline Issue Date & $2014-01-21$ \\
\hline oaire:version & VoR \\
\hline URL & https://hdl. handle. net/11094/82372 \\
\hline rights & $\begin{array}{l}\text { This article may be downloaded for personal use } \\
\text { only. Any other use requires prior permission } \\
\text { of the author and AIP Publishing. This article } \\
\text { appeared in The Journal of Chemical Physics } \\
140,034505(2014) \text { and may be found at } \\
\text { https://doi.org/10.1063/1.4861039. }\end{array}$ \\
\hline Note & \\
\hline
\end{tabular}

Osaka University Knowledge Archive : OUKA

https://ir. Library. osaka-u. ac. jp/

Osaka University 


\title{
Molecular dynamics analysis on wetting and interfacial properties of water-alcohol mixture droplets on a solid surface
}

\author{
D. Surblys, ${ }^{1, a)}$ Y. Yamaguchi, ${ }^{1, b)}$ K. Kuroda, ${ }^{2}$ M. Kagawa, ${ }^{2}$ T. Nakajima, ${ }^{2}$ and H. Fujimura ${ }^{2}$ \\ ${ }^{1}$ Department of Mechanical Engineering, Osaka University, 2-1 Yamadaoka, Suita 565-0871, Japan \\ ${ }^{2} R \&$ D Center, Dai Nippon Printing Co., Ltd., 1-1-3 Midorigahara, Tsukuba 300-2646, Japan
}

(Received 28 October 2013; accepted 19 December 2013; published online 16 January 2014)

\begin{abstract}
Molecular dynamics simulations of single water, water-methanol, or water-IPA (isopropyl-alcohol) mixture droplets on a solid surface were performed with various mixture ratios. An increase in alcohol fraction generally gave an increase in droplet wettability. Both methanol and IPA molecules showed a strong preference to gather at various interfaces, with methanol molecules also showing a tendency to diffuse into the droplet bulk. Specific interfacial tensions were investigated using quasi-one-dimensional simulation systems, and liquid-vapor and solid-liquid interfacial tensions were found to decrease greatly due to the presence of interfacial alcohol, while solid-vapor interfacial tensions were proved to have little influence on wettability. Young's relation was found to hold quantitatively well for both water-methanol and water-IPA droplets. The validity of using Bakker's equation on solid-liquid interfaces was also investigated, and it was shown that for tightly spaced crystal surfaces, the introduced uncertainly is small. () 2014 AIP Publishing LLC. [http://dx.doi.org/10.1063/1.4861039]
\end{abstract}

\section{INTRODUCTION}

Since the primary formulation by Young in 1805 , liquid behavior on solid surface, especially at three-phase interface including gas phase as well, has long been a topic of interest both as basic science and in various engineering fields due to its considerable practical importance. ${ }^{1-4}$ Particularly in recent industrial printing technology, the required resolution has reached up to nanometer scale in high-speed relief or gravure printing processes, and profound understanding of the contact behavior of a micro- or nano-scale liquid droplet on a solid surface has become essential for the control of ink motion and resulting printing quality.

Regarding microscopic aspect, Maruyama et al. ${ }^{5}$ carried out molecular dynamics (MD) simulations of a mono-atomic Lennard-Jones (L-J) liquid droplet on solid surface. They showed that the contact angle of the droplet had a simple first-order correlation with the potential well depth of the effective inter-atomic interaction between liquid and solid components, and they claimed that the macroscopic Young's equation could be extended to the molecular scale in this L-J system. Studies on wetting of L-J system have also been carried out, e.g., for molecular-level roughness of the surface, ${ }^{6}$ wetting of spherical particulates, ${ }^{7}$ or sessile droplet. $^{8}$ We have also performed MD simulations on wetting of a water droplet on an electrically polarized solid surface in order to investigate the effects of Coulomb interaction between liquid and solid components. ${ }^{9}$

Adding minute amounts of alcohol is a well-known way in various industrial fields to radically change the wetting behavior of water while the microscopic mechanism

\footnotetext{
a)Electronic mail: donatas@gcom.mech.eng.osaka-u.ac.jp.

b)Electronic mail: yamaguchi@mech.eng.osaka-u.ac.jp. URL: http://wwwgcom.mech.eng.osaka-u.ac.jp/ yamaguchi/.
}

has not been clearly understood yet, although some preliminary work has been done on investigating water-alcohol mixtures. ${ }^{10-12}$ In this study, we have performed MD simulations of water-methanol $\left(\mathrm{CH}_{3}-\mathrm{OH}\right)$ and water-IPA (isopropylalcohol: $\left.\left(\mathrm{CH}_{3}\right)_{2} \mathrm{CH}-\mathrm{OH}\right)$ droplets of various mixture ratios on a solid surface in order to investigate the effects of alcohol additives on droplet wettability. Specific values of each interfacial tension were also investigated by using separate quasione-dimensional systems. Finally, the validity of the method of obtaining the solid-liquid interfacial tensions was also examined.

\section{METHOD}

\section{A. Potential model}

Water $\left(\mathrm{H}_{2} \mathrm{O}\right)$, methanol $\left(\mathrm{MeOH}, \mathrm{CH}_{3}-\mathrm{OH}\right)$, and isopropyl-alcohol (IPA, $\left.\left(\mathrm{CH}_{3}\right)_{2} \mathrm{CH}-\mathrm{OH}\right)$ molecules, as shown in Fig. 1, are adopted as fluid components considering further comparison with our experiment and macroscopic simulations. ${ }^{13}$ The SPC/E ${ }^{14}$ and OPLS-UA ${ }^{15,16}$ potential models are applied for water and alcohol molecules, respectively, in this study. In the original OPLS-UA model, IPA molecules have one internal degree of freedom allowing rotation around the $\mathrm{O}-\mathrm{CH}$ bond. Instead of implementing this, IPA is represented by a combination of its three conformers in this study, as shown in Figs. 1(c) and 1(d) using the mixture ratios described in the original paper. ${ }^{16}$ This is done for the practical purpose of reducing the calculation cost, because with the internal degree of freedom the hydrogen atom with a small mass vibrates at a high rate, requiring a smaller time step. Indeed, we have also carried out simulations of droplet wetting using the original model with an internal degree of freedom, but no remarkable difference was observed between the results of the two models for several conditions. All 
(a)

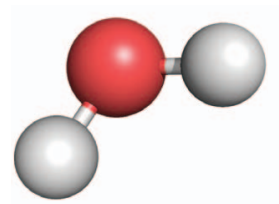

(c)
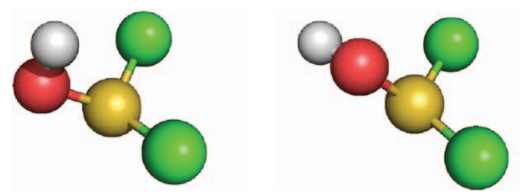

(d)

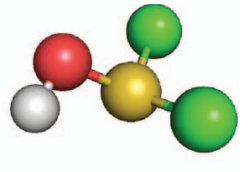

FIG. 1. Visual representations of molecules used in simulation. (a) Water molecule, (b) Methanol molecule, (c) IPA molecule trans conformer, and (d) IPA molecule gauche conformers.

molecules are treated as rigid bodies without intra-atomic degrees of freedom consisting of fixed interaction sites with constant masses, where $\mathrm{CH}_{3}, \mathrm{O}$, and $\mathrm{H}$ are set as the interaction sites for methanol and two $\mathrm{CH}_{3}, \mathrm{CH}, \mathrm{O}$, and $\mathrm{H}$ are set as the interaction sites for IPA. The 12-6 L-J and Coulomb potentials are assumed as the inter-molecular potentials between these sites, respectively, as in Eqs. (1) and (2):

$$
\begin{gathered}
\Phi^{\mathrm{LJ}}\left(r_{i j}\right)=H\left(r_{c}-r_{i j}\right) \cdot 4 \epsilon_{i j}\left[\left(\frac{\sigma_{i j}}{r_{i j}}\right)^{12}-\left(\frac{\sigma_{i j}}{r_{i j}}\right)^{6}\right. \\
\left.+\left\{c_{2}^{\mathrm{LJ}}\left(\frac{r_{i j}}{r_{c}}\right)^{2}-c_{0}^{\mathrm{LJ}}\right\}\right], \\
\Phi^{\mathrm{C}}\left(r_{i j}\right)=H\left(r_{c}-r_{i j}\right) \frac{q_{i} q_{j}}{4 \pi \epsilon_{0}}\left[\frac{1}{r_{i j}}+\frac{1}{r_{c}}\left\{c_{2}^{\mathrm{C}}\left(\frac{r_{i j}}{r_{c}}\right)^{2}-c_{0}^{\mathrm{C}}\right\}\right],
\end{gathered}
$$

where $r_{i j}$ is the distance between interaction sites $i$ and $j$, while $\epsilon_{i j}, \sigma_{i j}, q_{i}$, and $\epsilon_{0}$ denote the L-J energy and length parameters, point charge at site $i$, and vacuum permittivity, respectively. The L-J and Coulomb interactions in Eqs. (1) and (2) are truncated at a cut-off distance $r_{c}$ using the Heaviside step function $H$ and correction terms are also added so that the potentials approach zero smoothly at $r_{c}$ with the following coefficients:

$$
\begin{aligned}
& c_{2}^{\mathrm{LJ}}=6\left(\frac{\sigma_{i j}}{r_{c}}\right)^{12}-3\left(\frac{\sigma_{i j}}{r_{c}}\right)^{6}, \\
& c_{0}^{\mathrm{LJ}}=7\left(\frac{\sigma_{i j}}{r_{c}}\right)^{12}-4\left(\frac{\sigma_{i j}}{r_{c}}\right)^{6},
\end{aligned}
$$

$$
\begin{gathered}
c_{2}^{\mathrm{C}}=\frac{1}{2}, \\
c_{0}^{\mathrm{C}}=\frac{3}{2} .
\end{gathered}
$$

The cut-off distance $r_{c}$ is $1.5 \mathrm{~nm}$ in this study. There is no L-J interaction for single hydrogen sites, and the inter-L-J parameters for different components are determined by the Lorentz-Berthelot mixing rule, where for particles $i$ and $j$ the parameters are:

$$
\begin{aligned}
\sigma_{i j} & =\frac{\sigma_{i i}+\sigma_{j j}}{2}, \\
\epsilon_{i j} & =\sqrt{\epsilon_{i i} \cdot \epsilon_{j j}} .
\end{aligned}
$$

These mixing rules are used widely in MD simulations, and are applied to water-alcohol mixtures in numerous works. ${ }^{10,11,17-19}$ The (111) surface of an fcc crystal consisting of three layers is assumed as the solid wall. The inter-atomic potential between wall particles is expressed by the following harmonic potential for nearest neighbors:

$$
\Phi^{\mathrm{H}}\left(r_{i j}\right)=\frac{k}{2}\left(r_{i j}-r_{0}\right)^{2},
$$

where $r_{0}$ and $k$ denote the equilibrium distance and spring constant, respectively. The interactions between fluid and solid particles are expressed by the L-J potential and the interL-J parameters are also determined by the Lorentz-Berthelot mixing rule shown in Eqs. (7) and (8). The potential and mass

\begin{tabular}{|c|c|c|c|}
\hline$\sigma_{\mathrm{O}-\mathrm{O} \text { (water) }}(\mathrm{nm})$ & $\sigma_{\mathrm{O}-\mathrm{O}(\mathrm{MeOH}, \mathrm{IPA})}(\mathrm{nm})$ & $\sigma_{\mathrm{CH}_{3}-\mathrm{CH}_{3}(\mathrm{MeOH})}(\mathrm{nm})$ & $\sigma_{\mathrm{CH}_{3}-\mathrm{CH}_{3}(\mathrm{IPA})}(\mathrm{nm})$ \\
\hline 0.3166 & 0.307 & 0.3775 & 0.391 \\
\hline$\sigma_{\mathrm{CH}-\mathrm{CH}}(\mathrm{nm})$ & $\sigma_{\text {wall-wall }}{ }^{\mathrm{a}}(\mathrm{nm})$ & $\epsilon_{\mathrm{O}-\mathrm{O} \text { (water) }}(\mathrm{J})$ & $\epsilon_{\mathrm{O}-\mathrm{O}(\mathrm{MeOH}, \mathrm{IPA})}(\mathrm{J})$ \\
\hline 0.385 & 0.35 & $1.08 \times 10^{-21}$ & $1.182 \times 10^{-21}$ \\
\hline$\epsilon_{\mathrm{CH}_{3}-\mathrm{CH}_{3}(\mathrm{MeOH})}(\mathrm{J})$ & $\epsilon_{\mathrm{CH}_{3}-\mathrm{CH}_{3}(\mathrm{IPA})}(\mathrm{J})$ & $\epsilon_{\mathrm{CH}-\mathrm{CH}}(\mathrm{J})$ & $\epsilon_{\text {wall-wall }^{\mathrm{a}}(\mathrm{J})}$ \\
\hline $1.438 \times 10^{-21}$ & $1.112 \times 10^{-21}$ & $0.556 \times 10^{-21}$ & $1.44 \times 10^{-21}$ \\
\hline$q_{\mathrm{O} \text { (water) }}(\mathrm{e})$ & $q_{\mathrm{O}(\mathrm{MeOH}, \mathrm{IPA})}(\mathrm{e})$ & $q_{\mathrm{H} \text { (water) }}(\mathrm{e})$ & $q_{\mathrm{H}(\mathrm{MeOH}, \mathrm{IPA})}(\mathrm{e})$ \\
\hline-0.8476 & -0.7 & 0.4238 & 0.435 \\
\hline$q_{\mathrm{CH}_{3}(\mathrm{MeOH})}(\mathrm{e})$ & $q_{\mathrm{CH}(\mathrm{IPA})}(\mathrm{e})$ & $r_{0}(\mathrm{~nm})$ & $k(\mathrm{~N} / \mathrm{m})$ \\
\hline 0.265 & 0.265 & 0.277 & 46.8 \\
\hline
\end{tabular}
parameters are summarized in Tables I and II. The wall L-J energy parameter $\epsilon_{\text {wall-wall }}$ is empirically defined so that the contact angle of a pure water droplet becomes approximately $90^{\circ}$, and is only used for interaction between fluid and solid particles. Values of platinum crystal are adopted for the mass and lattice constants, Young's modulus and van der Waals radius, from which $r_{0}, \sigma_{\text {wall-wall, }}$ and $k$ are derived. A onedimensional wall potential is also created to mimic the mean

TABLE I. Potential parameters.

${ }^{a}$ Used only for Lorentz-Berthelot mixing rule. 
TABLE II. Mass parameters.

\begin{tabular}{lcccc}
\hline$m_{\mathrm{O}}(\mathrm{kg})$ & $m_{\mathrm{H}}(\mathrm{kg})$ & $m_{\mathrm{CH}_{3}}(\mathrm{~kg})$ & $m_{\mathrm{CH}}(\mathrm{kg})$ & $m_{\text {wall }}(\mathrm{kg})$ \\
\hline $2.657 \times 10^{-26}$ & $1.674 \times 10^{-27}$ & $2.497 \times 10^{-26}$ & $2.162 \times 10^{-26}$ & $3.239 \times 10^{-25}$ \\
\hline
\end{tabular}

potential field of a single layer of the previously described solid surface:

$$
\begin{aligned}
\Phi^{\mathrm{W}}\left(h_{i j}\right)= & H\left(r_{c}-h_{i j}\right) \frac{4 \pi \epsilon_{i j}}{r_{0}^{2} \sqrt{3}}\left[\left\{\frac{2}{5}\left(\frac{\sigma_{i j}}{h_{i j}}\right)^{12}-\left(\frac{\sigma_{i j}}{h_{i j}}\right)^{6}\right\}\right. \\
& \left.\times h_{i j}^{2}-c_{4}^{\mathrm{W}} h_{i j}^{4}+c_{2}^{\mathrm{W}} h_{i j}^{2}-c_{0}^{\mathrm{W}}\right],
\end{aligned}
$$

where $h_{i j}$ denotes the distance between site $i$ and plane $j$. The same L-J parameters are used as for the solid surface, and by placing there potential walls with $r_{0} \sqrt{\frac{2}{3}}$ interval, onedimensional solid surface potential field is reproduced. These walls only interact with fluid particles. The coefficients in Eq. (10) are as follows:

$$
\begin{gathered}
c_{4}^{\mathrm{W}}=\frac{1}{r_{c}^{2}}\left\{6\left(\frac{\sigma_{i j}}{r_{c}}\right)^{12}-3\left(\frac{\sigma_{i j}}{r_{c}}\right)^{6}\right\}, \\
c_{2}^{\mathrm{W}}=14\left(\frac{\sigma_{i j}}{r_{c}}\right)^{12}-8\left(\frac{\sigma_{i j}}{r_{c}}\right)^{6}, \\
c_{0}^{\mathrm{W}}=r_{c}^{2}\left\{\frac{42}{5}\left(\frac{\sigma_{i j}}{r_{c}}\right)^{12}-6\left(\frac{\sigma_{i j}}{r_{c}}\right)^{6}\right\} .
\end{gathered}
$$

\section{B. Simulation system}

Figure 2 exhibits the simulation system of a water-IPA mixture droplet on a solid surface, where a cylindrical shape was chosen to eliminate the effect of line tension on droplet

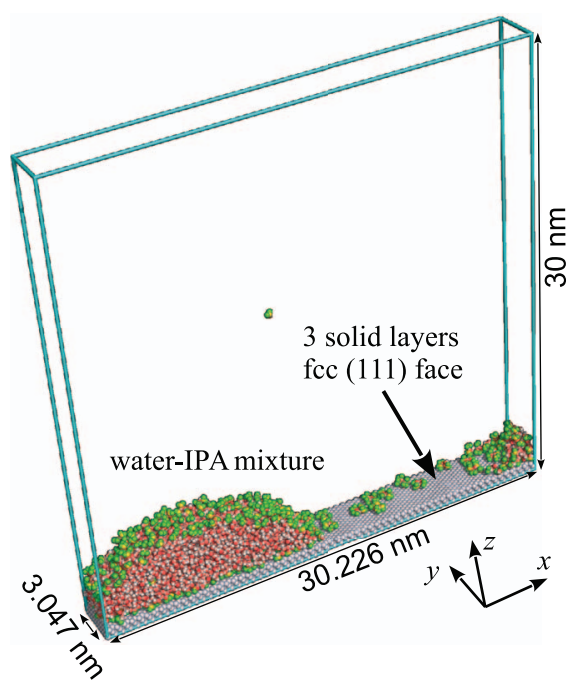

\begin{tabular}{|c|c|c|c|c|c|c|c|}
\hline $\begin{array}{l}\text { MeOH mass fraction } \\
f_{\mathrm{MeOH}}(\%)\end{array}$ & 0 & 8 & 15 & 21 & 26 & 31 & 35 \\
\hline No. of water molecules & 4000 & 4000 & 4000 & 4000 & 4000 & 4000 & 4000 \\
\hline No. of MeOH molecules & 0 & 200 & 400 & 600 & 800 & 1000 & 1200 \\
\hline
\end{tabular}

FIG. 2. Birds-eye view of the simulation system of a water-IPA mixture droplet on a solid surface. Simulations for a water-methanol mixture droplet are carried out in a system with the same size.
TABLE III. Compositions of water-methanol mixture droplets.

wettability. The three-layered solid surface is located at the bottom of the calculation cell with periodic boundary conditions in lateral $x$ - and $y$-directions and mirror boundary condition at the top boundary. The calculation region size is 30.226 $\times 3.047 \times 30.0 \mathrm{~nm}^{3}$ and simulations for a water-methanol mixture droplet are carried out in a system with the same size and boundary conditions. Droplet compositions with various methanol and IPA mass fractions, $f_{\mathrm{MeOH}}$ and $f_{\mathrm{IPA}}$, respectively, are shown in Tables III and IV. We have confirmed the constructed droplets are large enough that the results are not influenced by the radius range handled in this study, which is also reported in literature. ${ }^{20}$ The droplet systems are equilibrated for $8 \mathrm{~ns}$ and the average of $8 \mathrm{~ns}$ thereafter is used for analysis.

Additional simulation systems shown in Figs. 3 and 4 are created to quantitatively evaluate interfacial tensions, where the basic setup is the same as the droplet systems. Systems used to calculate solid-liquid and liquid-vapor interfacial tensions as in Fig. 3 have a calculation region size of 3.358 $\times 3.047 \times 21.0 \mathrm{~nm}^{3}$ and their compositions are shown in Tables V and VI. The average of simulation for $8 \mathrm{~ns}$ is used for analysis after prior equilibration of $8 \mathrm{~ns}$ for water-IPA systems and $16 \mathrm{~ns}$ for water-methanol systems. Systems used to calculate solid-vapor interfacial tensions as in Fig. 4 have a calculation region size of $19.191 \times 18.005 \times 12.0 \mathrm{~nm}^{3}$, and contain 10 to 300 IPA molecules without water molecules. No such systems are composed for methanol because the influence of solid-vapor interfacial tension on droplet wettability for water-IPA systems was negligibly small compared to that of solid-liquid and liquid-vapor tensions as described later. After an equilibration of $1 \mathrm{~ns}$, the average of $4 \mathrm{~ns}$ is used for analysis.

In all of the previously described systems, the position of solid atoms in the bottom layer is fixed and the temperature of those in the middle layer is controlled by the Langevin method ${ }^{21}$ at $298.15 \mathrm{~K}$ with a Debye temperature of $240 \mathrm{~K}$. The velocity Verlet method with modified quaternionconstraint $^{22,23}$ is applied for the integration of Newton's equation of motion with a time step $\Delta t$ of $1 \mathrm{fs}$.

One additional type of quasi-one-dimensional simulation systems shown in Fig. 5 is constructed to reassess the validity of solid-liquid interfacial tension calculation. Periodic boundary conditions are imposed in all lateral directions and

TABLE IV. Compositions of water-IPA mixture droplets.

\begin{tabular}{lrrrrrr}
\hline \hline IPA mass fraction $f_{\text {IPA }}(\%)$ & 0 & 8 & 14 & 20 & 25 & 29 \\
\hline No. of water molecules & 4000 & 4000 & 4000 & 4000 & 4000 & 4000 \\
No. of IPA molecules & 0 & 100 & 200 & 300 & 400 & 500 \\
\hline \hline
\end{tabular}




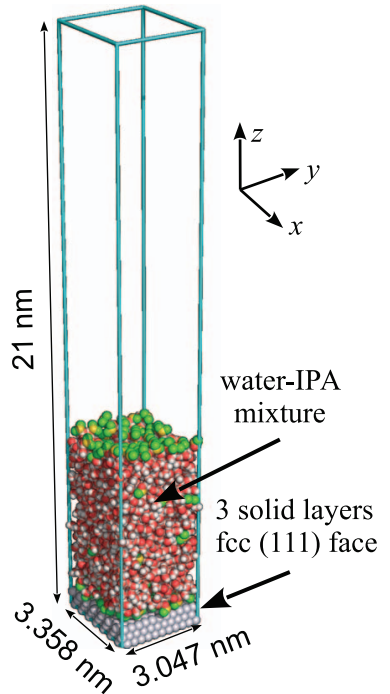

FIG. 3. Snapshot of a simulation system used to evaluate solid-liquid and liquid-vapor interfacial tensions for water-IPA mixture. The same system size is applied for water-methanol system.

the liquid inside is composed of either 3000 water, 1500 methanol, or 1000 IPA molecules. Two solid surfaces each containing three layers are symmetrically located at the center of the calculation cell, with innermost layers being $3 \mathrm{~nm}$ apart. The position of solid atoms in the innermost layers is fixed and the temperature of those in the middle layers is controlled by the Langevin method at $298.15 \mathrm{~K}$. In addition, two sets of three potential walls recreating the mean potential field of the solid surface are also placed symmetrically to the $x y$ plane. Thirty-seven simulation systems are created for each liquid type with the innermost potential planes being apart from 0 to $6 \mathrm{~nm}$. As well as the integration methods and temperature control used in the previous systems, additional temperature and pressure control is applied to the liquid phase with thermostat and barostat relaxation times $\tau_{T}$ and $\tau_{p}$ set to 2 and 5 ps, respectively, and pressure set to atmospheric value. ${ }^{24,25}$ The pressure control scheme is modified so that only the $z$-component of the local pressure tensor in the liquid phase is controlled as described in detail in the Appendix. The

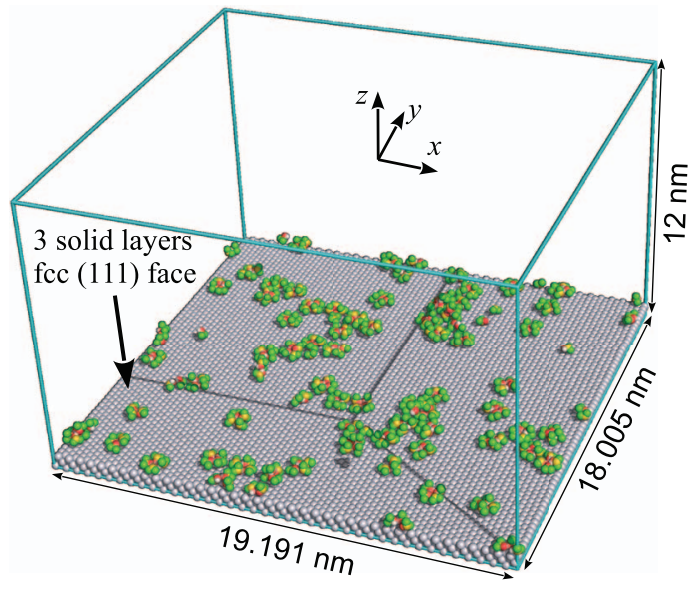

FIG. 4. Snapshot of a simulation system used to evaluate solid-vapor interfacial tension for IPA molecules.
TABLE V. Compositions of quasi-one-dimensional systems for watermethanol.

$\begin{array}{lllllllll}\text { No. of water molecules } & 1800 & 1800 & 1800 & 1800 & 1800 & 1800 & 1800 & 0\end{array}$

$\begin{array}{lllllllll}\text { No. of MeOH molecules } & 0 & 100 & 200 & 300 & 400 & 500 & 600 & 800\end{array}$

pressure control region starts at $0.175 \mathrm{~nm}$ outwards from the equilibrium position of the plane closest to the liquid phase, and no surface or liquid molecules are present at this region. The size of calculation region in $x$ - and $y$-directions is 3.358 $\times 3.047 \mathrm{~nm}^{2}$, while that in $z$-direction varies due to the pressure control scheme. After $1 \mathrm{~ns}$ of equilibration, the average of simulation for $8 \mathrm{~ns}$ data is used to analyze systems with the potential planes closest and furthest apart, while data of $2 \mathrm{~ns}$ are used for all others in-between.

\section{RESULTS AND DISCUSSION}

\section{A. Droplet contact angles}

Figures 6 and 7, respectively, display snapshots of water-methanol and water-IPA mixture droplets with different alcohol mass fractions on solid surfaces and corresponding two-dimensional density distributions of alcohol molecules. The total number of water molecules is kept unchanged as indicated in Tables III and IV, and the density distribution is calculated around the center of mass of the droplet by assuming a symmetric shape.

With a low methanol concentration of $f_{\mathrm{MeOH}}=8 \%$ shown in Fig. 6(a), methanol molecules have the highest concentration at the three phase interface line and tend to gather mostly at the solid-liquid interface and to a lesser extent at the liquid-vapor interface. At a methanol concentration of $f_{\mathrm{MeOH}}=21 \%$ in Fig. 6(b), even more alcohol molecules gather at the solid-liquid interface and form a clear methanol mono-layer. At a higher methanol concentration of $f_{\mathrm{MeOH}}$ $=35 \%$ in Fig. 6(c), a large amount of methanol molecules diffuse into the droplet bulk, showing good solubility.

On the other hand, the system with a low IPA concentration of $f_{\text {IPA }}=8 \%$ shown in Fig. 7(a) has IPA molecules gathering at solid-liquid and solid-vapor interfaces, and this feature is similar to the water-methanol mixture system with a low methanol concentration shown in Fig. 6(a). However, the tendency to gather at the three phase interface line is much stronger for IPA molecules. In addition, an IPA mono-layer can be observed partly extending from the three phase interface towards the solid-liquid interface. With higher IPA concentrations of $f_{\mathrm{IPA}}=20 \%$ and $f_{\mathrm{IPA}}=29 \%$ in Figs. 7(b) and 7(c), more alcohol molecules gather at the solid-liquid and liquid-vapor interfaces and a distinct IPA mono-layer is formed at the solid-liquid interface. It is remarkable that even at very high concentrations IPA molecules do not diffuse inside the bulk, but rather begin to cover the

TABLE VI. Compositions of quasi-one-dimensional systems for water-IPA.

\begin{tabular}{llllllllll}
\hline \hline No. of water molecules & 1800 & 1800 & 1800 & 1800 & 1800 & 1800 & 1800 & 0
\end{tabular}

$\begin{array}{lllllllll}\text { No. of IPA molecules } & 0 & 50 & 60 & 70 & 80 & 90 & 100 & 500\end{array}$




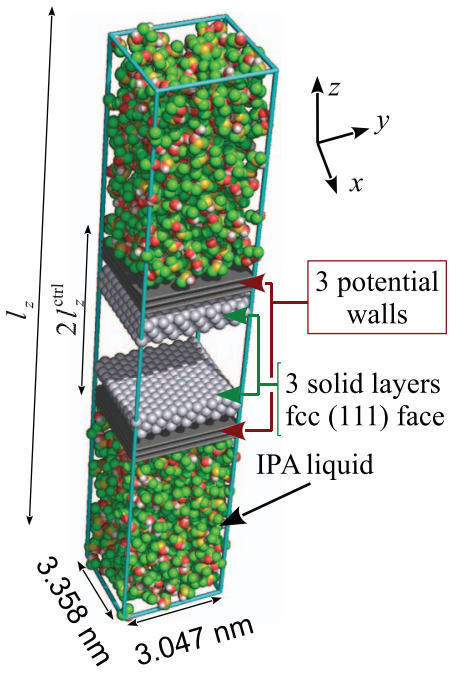

FIG. 5. Snapshot of single-component IPA simulation system used to reassess the validity of solid-liquid tension calculation. Cell size in the $z$ dimension $l_{z}$ varies due to the pressure control scheme, and $l_{z}^{\text {ctrl }}$ indicates the distance of the pressure control region from the system center, with details given in the Appendix. The same system dimensions are also used for single-component water and methanol simulations.

solid-vapor interface, as can be observed in Fig. 7(c), thus creating a very different system compared to that of watermethanol mixture droplets. This difference in solubility is thought to come from the large hydrophobic group of IPA compared to that of methanol, which means that more hydro-
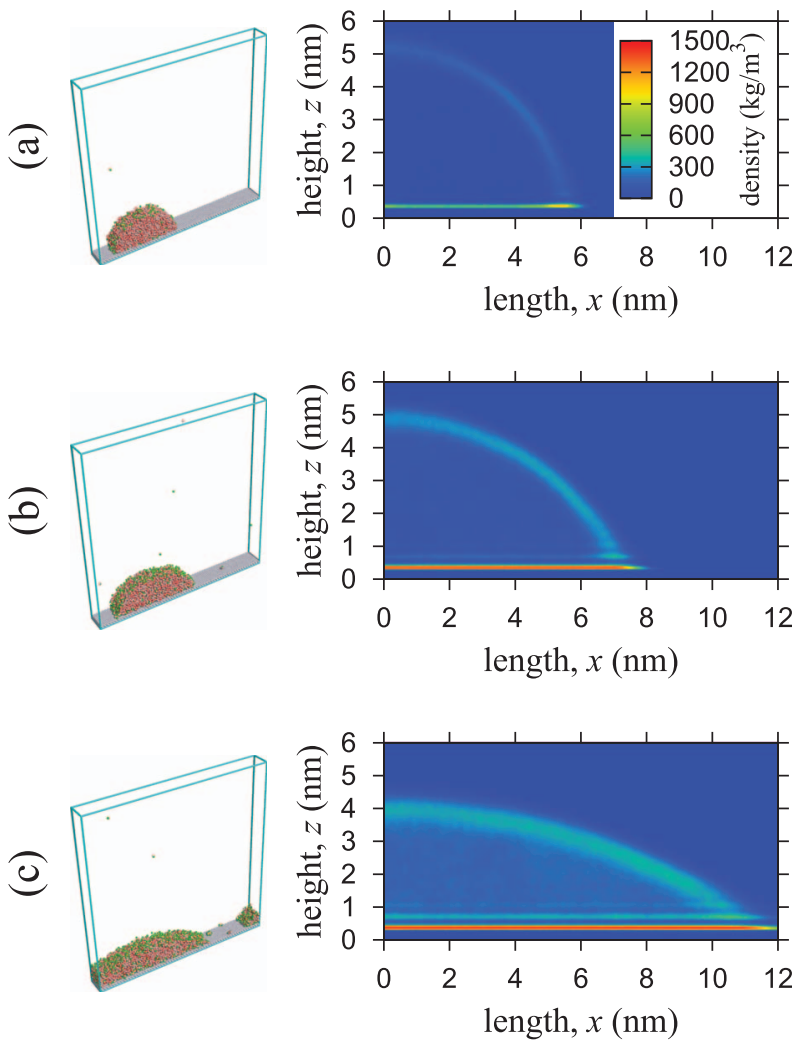

FIG. 6. (Left) Snapshots of water-methanol mixture droplet on a solid surface with different methanol fractions $f_{\mathrm{MeOH}}$, and (Right) two-dimensional distributions of methanol density from the center of mass of the droplet. (a) $f_{\mathrm{MeOH}}=8 \%$, (b) $f_{\mathrm{MeOH}}=21 \%$, and (c) $f_{\mathrm{MeOH}}=35 \%$.
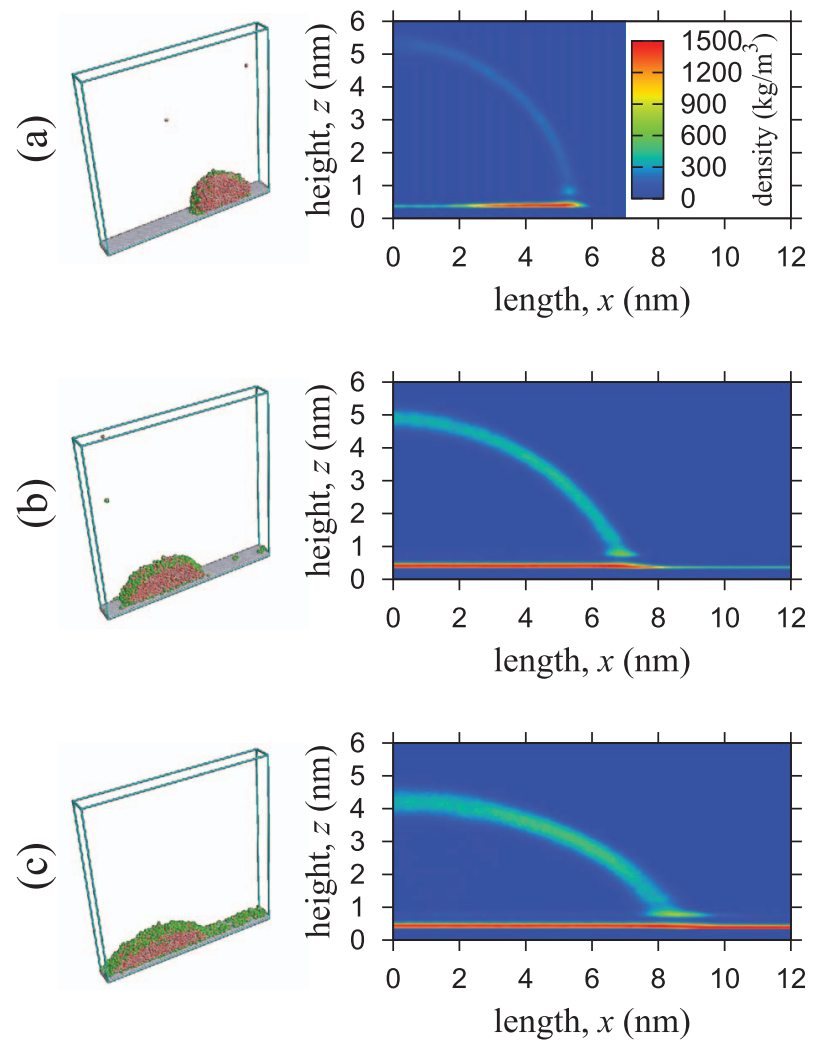

FIG. 7. (Left) Snapshots of water-IPA mixture droplet on a solid surface with different IPA fractions $f_{\text {IPA }}$, and (Right) two-dimensional distributions of IPA density from the center of mass of the droplet. (a) $f_{\mathrm{IPA}}=8 \%$, (b) $f_{\mathrm{IPA}}=20 \%$, and (c) $f_{\mathrm{IPA}}=29 \%$.

gen bonds have to be broken to accommodate IPA molecules inside water bulk.

The tendency for alcohol molecules to gather at solidliquid and liquid-vapor interfaces can be easily explained by the fact that methanol and IPA have lower liquid-vapor and, as is shown later, solid-liquid interfacial tensions than water. Because of this, it is energetically advantageous for alcohol molecules to gather at the interfaces and the resulting droplet energy is lowered. The same logic can also be applied to the three-phase interface. Even though it has no effect on the wettability of a cylindrical droplet, a distinct line tension exists at the three-phase interface. The increased alcohol density there indicates that the energy decrease due to alcohol additives at the three-phase interface might be initially larger than those at solid-liquid and liquid-vapor interfaces.

An apparent contact angle is measured by fitting a circle to a density contour line and measuring its angle against a plane elevated above the equilibrium position of the topmost solid surface layer. The density and elevation are $745 \mathrm{~kg} / \mathrm{m}^{3}$ and $0.365 \mathrm{~nm}$ for water-methanol, and $876 \mathrm{~kg} / \mathrm{m}^{3}$ and $0.294 \mathrm{~nm}$ for water-IPA droplets, respectively. These density and elevation values are obtained in quasi-one-dimensional systems shown in Fig. 3 in which the positions of solid-liquid and liquid-vapor interfaces can be determined from the balance of force and momentum due to pressure tensor distribution..$^{20}$ Details on how to obtain the required local pressure tensor are given in Sec. III B. The relation between alcohol mass fraction in mixture droplets and the cosine 


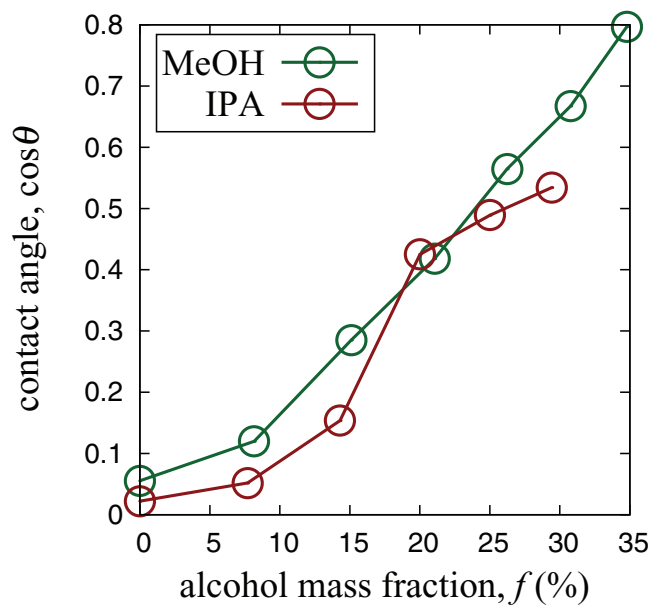

FIG. 8. Relation between the cosine of contact angle of water-alcohol mixture droplet and alcohol mass fraction.

of their contact angle $\theta$ is illustrated in Fig. 8 for both water-methanol and water-IPA droplets. An obvious increase in wettability with smaller contact angle is observed with increasing alcohol fractions in both water-methanol and waterIPA mixture droplets. The density distributions in Figs. 6 and 7 suggest that the change in contact angle is not simply due to a decrease in liquid-vapor surface tension, but also due to a significant change in the solid-liquid interfacial energy. Both water-methanol and water-IPA mostly show a substantial increase in $\cos \theta$ with the increase of alcohol fractions $f_{\mathrm{MeOH}}$ and $f_{\mathrm{IPA}}$, however, the increase seems almost linear for watermethanol systems, while water-IPA systems show a more irregular behavior. Specifically, $\cos \theta$ at an IPA mass fraction of $f_{\mathrm{IPA}}=8 \%$ changes very little from that at $f_{\mathrm{IPA}}=0 \%$. This is thought to be because the IPA mono-layer is only formed in proximity to the three phase interface and there is very little amount of IPA molecules in liquid-vapor and solid-vapor interfaces. Once the IPA mono-layer is formed, however, any extra IPA molecules are free to occupy other interfaces and influence wettability. Because of this, both methanol and IPA molecules seem to enhance wettability to similar amounts at larger mass fractions, although IPA starts to show signs of saturation at higher fractions of $f_{\mathrm{IPA}}>20 \%$.

\section{B. Interfacial tensions}

Each interfacial tension is evaluated using quasi-onedimensional systems: liquid-vapor and liquid-solid interfacial tensions from systems as in Fig. 3, and solid-vapor interfacial tension from systems as shown in Fig. 4. According to Bakker's equation, the interfacial tension is obtained by integrating local pressure tensor components along the direction normal to a flat interface ${ }^{26}$

$$
\gamma=\int\left(p_{N}-p_{T}\right) \mathrm{d} z
$$

where $\gamma$ is the interfacial tension and $p_{N}$ and $p_{T}$ are normal and tangential pressures to the interface, respectively. It should be noted that for the solid-liquid interface, the integration region is limited to the area containing liquid molecules, even though the pressure tensor has values even in areas where no molecules exist. A common practice not to include the contribution from interactions between the solid surface atom and liquid molecule when calculating tangential pressure $p_{T}$ and this convention is also followed in this work. ${ }^{27}$ Systems are divided into slabs along the $z$-axis and local pressure tensor is calculated by using weighted virial pressure. ${ }^{28}$ Strictly speaking, unlike the liquid-vapor interfacial tension, the solid-liquid and solid-vapor interfacial tensions obtained by Eq. (14) are not absolute values, but "relative" to those of a solid-vacuum interface. The implication of this is discussed later in this section.

While methanol fraction inside liquid bulk $f_{\mathrm{MeOH}}^{\text {bulk was }}$ proved to be a sufficient parameter to determine the state of each interface, relative IPA adsorption was chosen to express the state of each interface for water-IPA mixture droplets because the IPA molecules do not diffuse into droplet bulk as shown in the density distribution in Fig. 7 and its fraction inside liquid bulk is not an appropriate parameter to express the interface state. The relative IPA adsorption for an interface between $\alpha$ and $\beta$ phases $\Gamma_{\text {IPA }}^{\alpha \beta}$ against an arbitrary Gibbs dividing surface is given by

$$
\Gamma_{\text {IPA }}^{\alpha \beta}=N_{\text {IPA }}^{\alpha \beta}-N_{\text {water }}^{\alpha \beta} \frac{n_{\text {IPA }}^{\beta}-n_{\text {IPA }}^{\alpha}}{n_{\text {water }}^{\beta}-n_{\text {water }}^{\alpha}},
$$

where $n$ and $N$ are number density and surface excess per unit area for each substance in each phase. Relative adsorption is an invariant independent of the location of the Gibbs dividing surface. For water-methanol systems at high methanol concentrations, the alcohol molecules at interfaces and that inside liquid bulk increase at a similar rate, producing close relative adsorption values for different systems. Because of this, mass fraction inside liquid bulk $f_{\mathrm{MeOH}}^{\text {bulk }}$ is used for water-methanol systems.

Solid-liquid and liquid-vapor interfacial tensions $\gamma^{s l}$ and $\gamma^{l v}$ for water-methanol and water-IPA mixtures are displayed in Figs. 9 and 10, in which methanol bulk fraction $f_{\mathrm{MeOH}}^{\text {bulk and }}$ relative IPA adsorption $\Gamma_{\text {IPA }}$ at each interface are respectively adopted as parameters. The change of $\gamma_{\mathrm{MeOH}}^{s l}, \gamma_{\mathrm{MeOH}}^{l v}$, and $\gamma_{\mathrm{IPA}}^{l v}$, respectively, shown in Figs. 9(a), 9(b), and 10(b) is gradual and each seems to approach the value of single-component
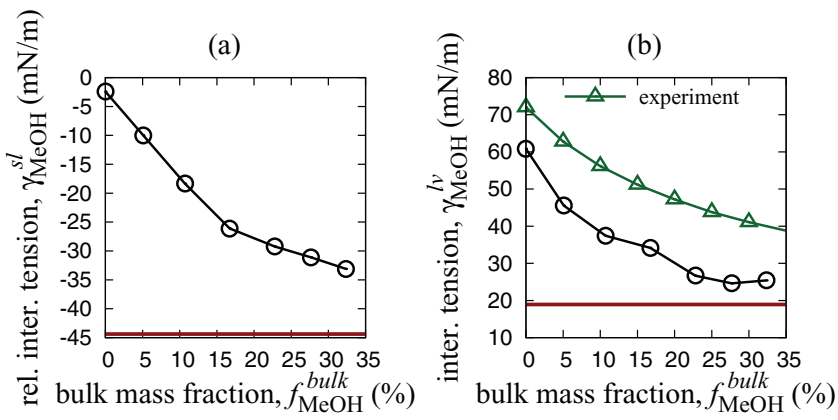

FIG. 9. Dependence of interfacial tensions for water-methanol mixtures on methanol bulk fraction evaluated in quasi-one-dimensional systems. Red horizontal lines show the values of single-component methanol. Experimental values are also included for liquid-vapor interfacial tensions. ${ }^{33}$ Note that the values provided for the solid-liquid interface are relative to that of solidvacuum. (a) Solid-liquid and (b) Liquid-vapor. 

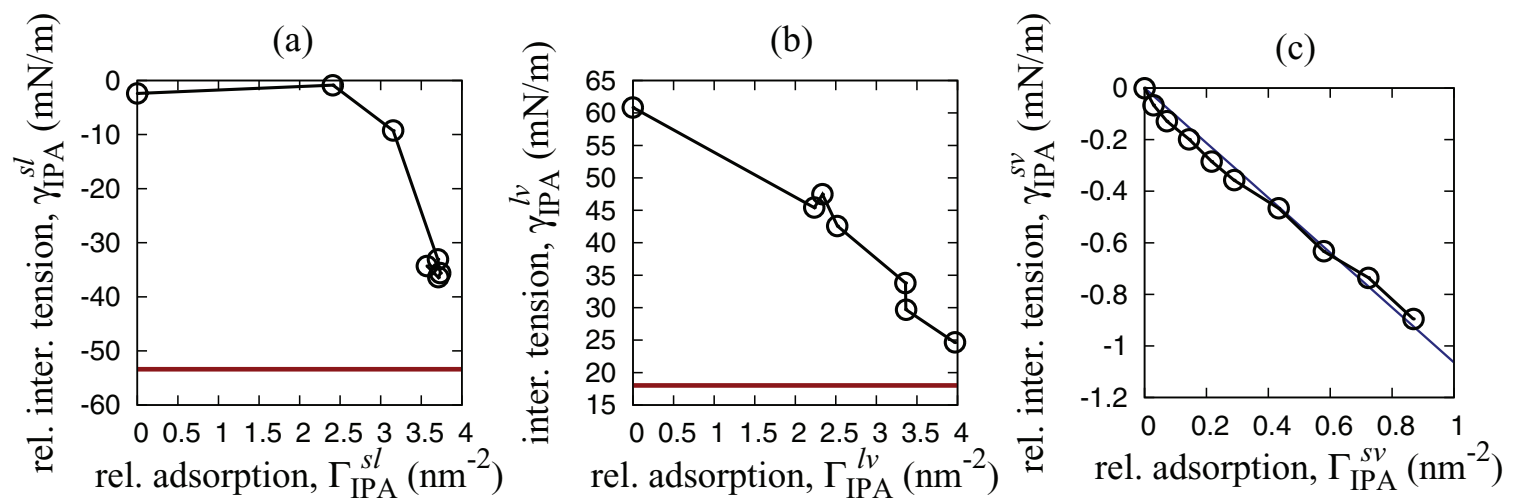

FIG. 10. Dependence of interfacial tensions in water-IPA mixtures on the relative IPA adsorption at each interface evaluated in quasi-one-dimensional systems. Red horizontal lines show the values of single-component IPA. A linear fitting is displayed for solid-vapor interfacial tension with a blue line, where the fitting function is $\gamma_{s v}=\zeta \cdot \Gamma_{\text {IPA }}^{s v}$ with $\zeta=-1.06 \times 10^{-21}(\mathrm{Nm})$. Note that the values provided for the solid-liquid and solid-vapor interfaces are relative to that of solid-vacuum. (a) Solid-liquid, (b) Liquid-vapor, and (c) Solid-vapor.

alcohol at higher solvent amounts. There is some irregularity seen in Fig. 10(b), which is thought to come from the IPA molecules at the interface which are trapped at a local minimum configuration that is different from the other systems, and this might occur easier for IPA because of large molecular size compared to the cross section area of the system. Experimental values are also included for $\gamma_{\mathrm{MeOH}}^{l v}$ in Fig. 9(b) for reference, and show that the simulation values are lower by up to $15 \mathrm{mN} / \mathrm{m}$ because the SPC/E and OPLS-UA potentials are not optimized to reproduce interfacial tensions. Nonetheless, the curves are qualitatively similar, and the wetting phenomenon is thought to be essentially the same.

A very steep decrease in the relative solid-liquid interfacial tension $\gamma_{\mathrm{IPA}}^{s l}$ of water-IPA mixture is observed in Fig. 10(a), and this is thought to be due to the formation of a complete IPA mono-layer on the solid-liquid interface. The four overlapping data points indicate that the mono-layer has a certain preferable number density and interfacial tension, thus the other data points show an unnatural state only possible in quasi-one-dimensional systems. Indeed, a fully formed high-density mono-layer can be observed extending from the three phase interface in Fig. 7(a). Although a solidliquid mono-layer also exists in water-methanol systems, its formation is less restrictive due to higher bulk solubility, and no such extreme changes in interfacial tensions are observed.

The relative solid-vapor interfacial tension $\gamma_{\mathrm{IPA}}^{s v}$ shown in Fig. 10(c) is almost linearly correlated to the IPA adsorption amount, and this is presumably because the number of hydrogen bonds created in the solid-vapor interface also linearly depends on $\Gamma_{\mathrm{IPA}}^{s v}$. It should be noted that the relative solidvapor interfacial tension has a much smaller absolute value, and is not thought to play an important role in determining the contact angle, i.e., wettability. Because of this and also as methanol molecules do not show any preference to moisten the surface, no further evaluation of the solid-vapor interfacial tension with methanol molecules is carried out in this study.

The cause and meaning of the negative values of relative solid-liquid and solid-vapor interfacial tensions should also be addressed here. Because the interaction between solid particles is modeled by the harmonic potential in which the interaction pairs are prescribed a priori to connect the near- est neighbors and zero point of the potential energy is set at an equilibrium distance, the total potential energy of a crystal solid surface without thermal vibrations placed in vacuum is zero, and solid bulk has no energetic advantage over surface in this model, even though the particles in solid bulk have more interaction pairs than those at the surface. If liquid or vapor molecules are adsorbed onto this solid surface, the total potential energy will decreases from zero because this is energetically preferable to vacuum since the L-J potential with a negative well is used for solid-liquid interactions. This means that the solid-liquid and solid-vapor interfacial tensions calculated by the Bakker's equation are relative ones to vacuum and apparently have negative values due to the zero-point setting of the potential model. Since only the difference between the solid-liquid and solid-vapor interfacial tensions has influence on wettability, as shown in the Young's relation of Eq. (16) in Sec. III C, relative values are enough for the two. On the other hand, the liquid-vapor interfacial tension calculated here is the absolute one which is always positive.

\section{Applicability of Young's equation}

An equation describing the interfacial tension balance at the three phase interface was derived by Young ${ }^{29}$

$$
\gamma^{s l}-\gamma^{s v}+\gamma^{l v} \cos \theta=0
$$

and this gives a way to predict the droplet contact angle from interfacial tensions calculated in Sec. III B.

Alcohol fractions in liquid bulk region in a droplet and relative alcohol adsorption amounts at each interface are calculated for the water-methanol and water-IPA droplet systems and the results are shown in Fig. 11. The alcohol fractions in Fig. 11(a) reflect the observations made from Figs. 6 and 7 in Sec. III A that methanol molecules dissolve into the droplet bulk much easier than IPA molecules. In addition, the relative adsorption amounts of each interface in Figs. 11(b) and 11(c) reveal that the solid-liquid interface in a waterIPA droplet at $f_{\mathrm{IPA}}>15 \%$ is saturated by the creation of a mono-layer and excess alcohol molecules start to overflow into the solid-vapor interface, while no such distinct signs of saturation can be observed for water-methanol droplets. Some 


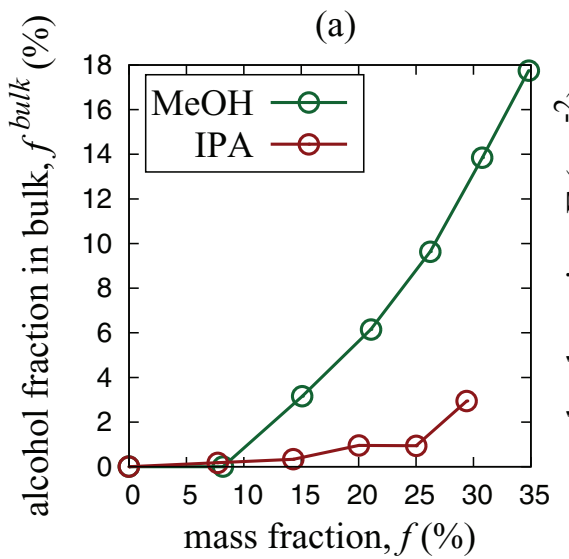

(b)

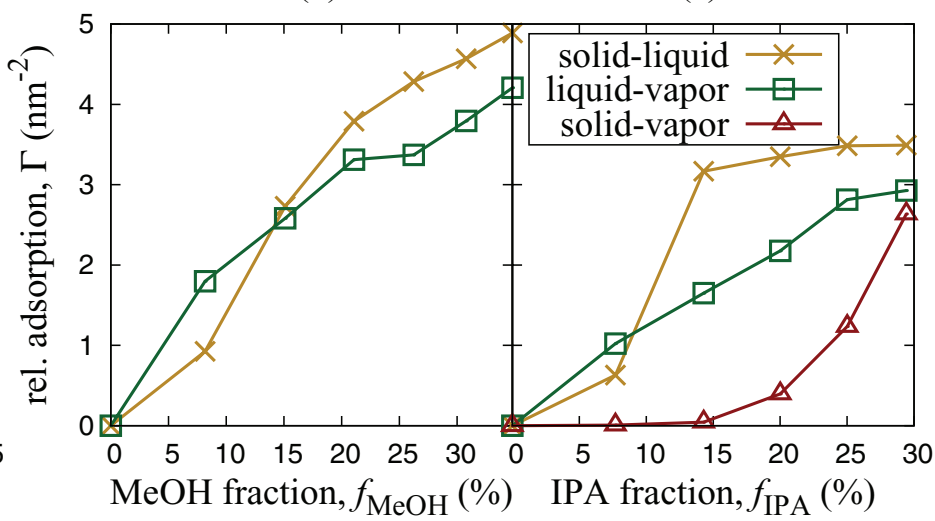

FIG. 11. (a) Methanol and IPA fractions in liquid bulk in relation to the overall mass fractions in droplet systems. (b) Water-methanol and (c) water-IPA relative adsorption amounts at each droplet interface. The IPA fraction in liquid bulk and methanol relative adsorption amounts are not used in determining droplet interfacial tensions and are only displayed for reference.

of our preliminary results indicate that the interface also saturates for water-methanol mixtures at a high enough alcohol ratio, but as methanol readily dissolves into water, this only means that the increase rates of interfacial and bulk alcohol become similar, describing a completely different state from the saturation seen in water-IPA.

Interfacial tensions are estimated by linear interpolation of the data shown in Figs. 9, 10(a), and 10(b) for solid-liquid and liquid-vapor interfaces or by the linear fitting function shown in Fig. 10(c) for relative solid-vapor interfacial tension with IPA. The relative solid-vapor interfacial tension for water-methanol mixture droplets is assumed to be always zero.

The predicted contact angles are compared with the measured ones in Fig. 12. Although there are some discrepancies, a good quantitative agreement can be seen. This means that our method accurately predicts interfacial tensions and shows that Young's approach is still applicable even at nano-scale.

\section{Reassessment of interfacial tension measuring}

Bakker's equation was used to evaluate interfacial tensions in Sec. III B under the assumption that the interfaces cannot support elastic strain. ${ }^{26}$ This is not the case, however, for solid-liquid and solid-vapor interfaces where the solid molecules at the surface are allowed to vibrate around their equilibrium position, and thus can indeed support strain. ${ }^{30}$ This results in the following Shuttleworth equation:

$$
s=\gamma+\frac{\mathrm{d} \gamma}{\mathrm{d} \varepsilon}
$$

where $s$ and $\varepsilon$ are interfacial stress and elastic strain, respectively. Because of this, using Bakker's equation on solidliquid and solid-vapor interfaces is appropriate only when the solid surface is completely periodic or a potential wall, such as described by Eq. (10). An alternative measuring way is needed to accurately evaluate the interfacial tension when the second term in the right-hand side of Eq. (17) is non-zero. Thermodynamic integration proposed by Leroy et al. ${ }^{31}$ is selected for this purpose. The basic premise is to use a potential wall in a similar way to a piston to quasi-reversibly push the fluid away from the solid surface by moving the said potential wall. The reversible work done by the potential wall corresponds to the difference of Gibbs free surface energy between the walls and the thermodynamic work done to increase the system volume. Several systems as shown in Fig. 5 are prepared in which the potential walls are placed at various positions. Only single-component liquid systems are used (a)

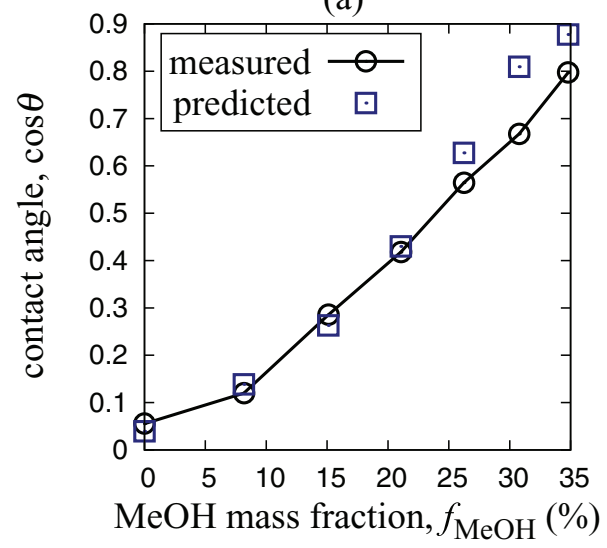

(b)

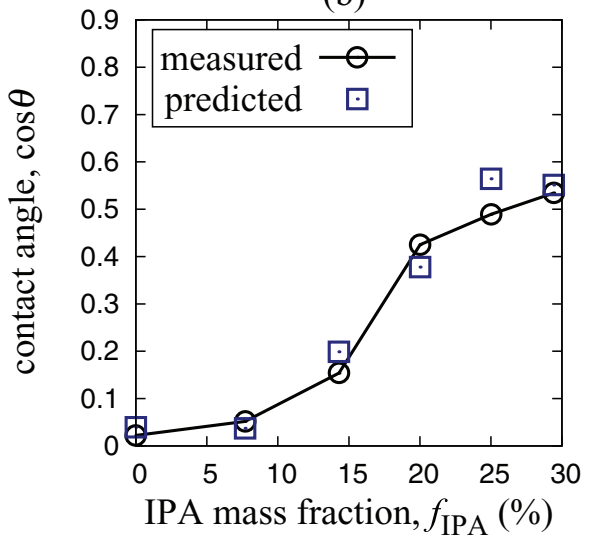

FIG. 12. Relation between the cosine of contact angle of the water-alcohol mixture droplets and alcohol mass fractions. (a) Water-methanol and (b) Water-IPA. 
(a)

(b)

(c)

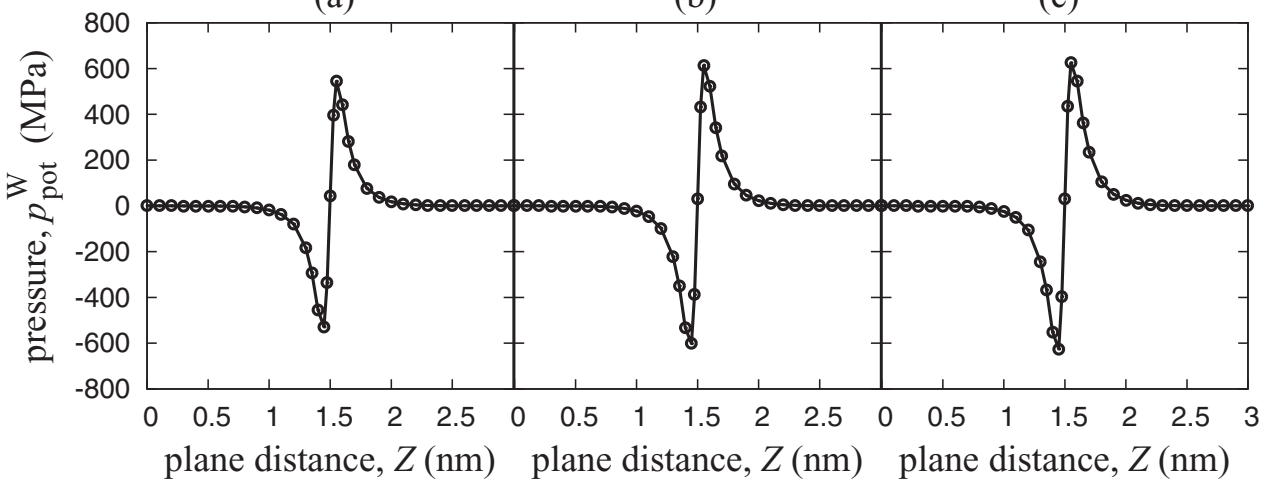

FIG. 13. Relation between pressure exerted on the potential walls and distance between innermost potential wall and the center of the system shown in Fig. 5 . The two solid walls are positioned $1.5 \mathrm{~nm}$ from the center of the calculation cell. (a) Water, (b) Methanol, and (c) IPA.

since in mixtures the chemical potential and interfacial adsorption must also be taken into account. The difference in the solid-liquid interfacial tension between different walls is

$$
\gamma_{\mathrm{solid}}^{s l}-\gamma_{\mathrm{pot}}^{s l}=-\int_{Z^{0}}^{Z^{1}} p_{\mathrm{pot}}^{\mathrm{W}} \mathrm{d} Z+\frac{p_{N}}{2}\left(l_{z}^{1}-l_{z}^{0}\right),
$$

where $\gamma_{\text {solid }}^{s l}$ and $\gamma_{\text {pot }}^{s l}$ are the relative solid-liquid interfacial tensions for solid surface and potential wall, while $p_{\text {pot }}^{\mathrm{W}}$ is the pressure exerted on the potential walls by liquid molecules. The distance from the center of the calculation cell to the innermost potential plane and the mean length of calculation cell in $z$-direction are, respectively, denoted by $Z$ and $l_{z}$, and the values of $Z$ before and after the quasi-reversible change are $Z^{0}=0 \mathrm{~nm}$ and $Z^{1}=3 \mathrm{~nm}$. It should be noted that the potential walls are constructed to accurately represent the mean potential field of the solid surfaces.

The pressure exerted on the potential walls is shown in Fig. 13. All of the graphs show a similar trend with the pressure being negative at $Z<1.5 \mathrm{~nm}$ and positive at $Z>1.5 \mathrm{~nm}$, i.e., potential walls are attracted towards the liquid components at $Z<1.5 \mathrm{~nm}$, and pushed back by the liquid components at $Z>1.5 \mathrm{~nm}$. The peaks of water component are slightly smaller than those of methanol and IPA, showing a weaker interaction between water and solid surface than that between alcohol and solid surface. This is to be expected, since the absolute value of the relative solid-liquid interfacial tension of water is much smaller than that of either methanol or IPA, as can be seen in Figs. 9 and 10, respectively.

In addition to the difference in Gibbs free energy given by Eq. (18), Bakker's equation, shown in Eq. (14), is used to directly calculate interfacial tensions $\gamma_{\text {Bakker }}^{s l}$ and $\gamma_{\text {pot }}^{s l}$ at $Z=0 \mathrm{~nm}$ and $Z=3 \mathrm{~nm}$, respectively, where $\gamma_{\text {Bakker }}^{s l}$ is the value obtained by using Bakker's equation directly on the solid surface and is thought to contain error. The results are summarized in Table VII. It is interesting to note that solid surfaces have lower solid-liquid interfacial tensions than potential walls for all liquids. This is to be expected, since the solid surface has a structure which allows liquid molecules to take more energetically stable configurations, and that should result in decreasing the interfacial energy compared to a flat potential wall. This is also confirmed by the fact that the difference is greater for water and methanol with smaller molecule size than IPA. The interfacial tensions obtained by Bakker's equation and those obtained by thermal integration only differ by about $2.6 \mathrm{mN} / \mathrm{m}$ at most. This is because the solid surface used in this work is a perfect fcc crystal with only minor thermal vibration and a small lattice spacing compared to the size of liquid molecules, and therefore approximating it as a flat potential wall does not produce a large error. This is effectively done by the Bakker's integration with only using solid-liquid interaction contribution to normal pressure as already described in Sec. III B. It is expected that the error would be much larger for rough or amorphous surfaces.

By comparing the interfacial tensions obtained by directly using Bakker's equation $\gamma_{\text {Bakker }}^{s l}$ and thermal integration $\gamma_{\text {solid }}^{s l}$, it is possible to roughly approximate the margin of error introduced by using Bakker's equation on a solid-liquid interface. For water and methanol systems, thermal integration produced lower interfacial tensions, and the methanol data showing a larger discrepancy was chosen to approximate the margin of error for water-methanol systems. On the other hand, thermal integration for IPA systems produced higher interfacial tensions, thus both water and IPA data were used for water-IPA systems to give an error range in both directions. The results are displayed in Fig. 14. The error bars show that although of similar order, the uncertainty brought by the Bakker's equation does not explain the discrepancies between measured and predicted contact angles.

In this work, only the uncertainty for the prediction of solid-liquid interfacial tension is investigated, but that for liquid-vapor interfacial tension should also be considered. Specifically, it is a well known fact that liquid-vapor interfacial tension is influenced by the droplet curvature, ${ }^{20}$ and this is especially remarkable for very small droplets, such as in

TABLE VII. The difference in Gibbs free surface energy of solid-liquid interface between solid surface and potential wall together with interfacial tensions obtained using various methods.

\begin{tabular}{lccr}
\hline \hline & Water & Methanol & \multicolumn{1}{c}{ IPA } \\
\hline$\gamma_{\text {solid }}^{s l}-\gamma_{\text {pot }}^{s l}(\mathrm{mN} / \mathrm{m})$ & -2.37 & -1.66 & -0.92 \\
$\gamma_{\text {pot }}^{s l}(\mathrm{mN} / \mathrm{m})$ & -1.12 & -46.15 & -51.97 \\
$\gamma_{\text {solid }}^{s l}(\mathrm{mN} / \mathrm{m})$ & -3.50 & -47.81 & -52.89 \\
$\gamma_{\text {Bakker }}^{s l}(\mathrm{mN} / \mathrm{m})$ & -2.16 & -45.22 & -53.97 \\
\hline \hline
\end{tabular}


(a)

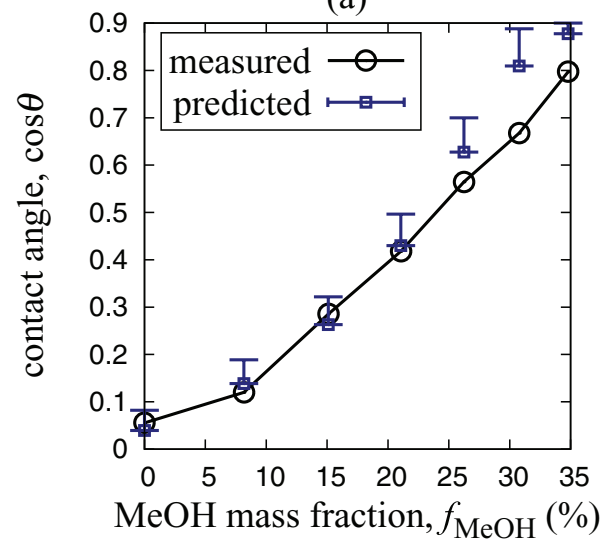

(b)

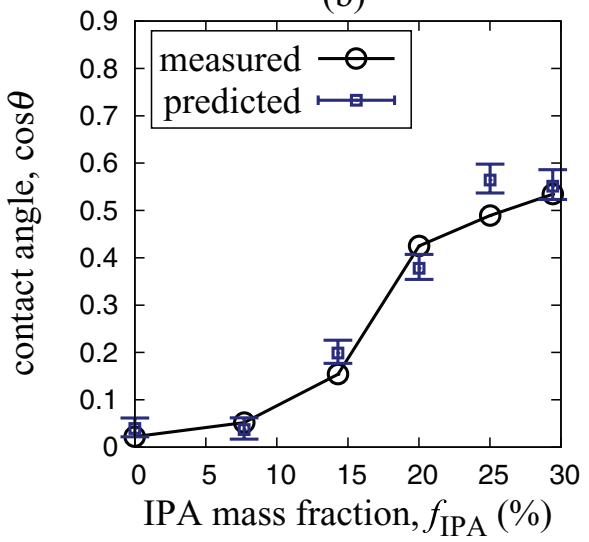

FIG. 14. Relation between the cosine of contact angle of the water-alcohol mixture droplets and alcohol mass fraction. Error bars show the uncertainty incurred by using Bakker's equation on a solid-liquid interface. (a) Water-methanol and (b) Water-IPA.

this paper. The current method of using flat interfaces does not take this into account and therefore some degree of error is expected, although some preliminary research has been done to ensure that the error is relatively small and does not affect the results anymore than the uncertainty coming from the solid-liquid interfacial tension.

\section{CONCLUDING REMARKS}

MD simulations of single water-methanol and water-IPA mixture cylindrical droplets were performed to investigate the effect alcohol additives have on water droplet wettability. The droplets generally became more wettable with larger alcohol fractions. Both types of alcohol molecules showed a strong preference to gather at solid-liquid and liquid-vapor interfaces, and a clear tendency to gather at the three-line interface was also observed at very low mixture ratios. At high mixture ratios, methanol molecules diffused well into the droplet bulk, while IPA molecules were found to spread out to the solid-vapor interface with almost no molecules dissolving inside the liquid droplet, thus creating two very different mixture systems.

Specific interfacial tensions were calculated in quasi-one-dimensional simulation systems and it was also quantitatively confirmed that alcohol greatly decreases liquid-vapor and solid-liquid interfacial tensions, while very little change occurs in the solid-vapor interface. Droplet interfacial tensions were assessed using data obtained in the quasi-one-dimensional systems and gave good quantitative estimation of the contact angle based on Young's equation, indicating that the approach of interfacial tension balance at the three-phase interface is still valid at nano-scale.

The validity of using Bakker's equation on solid-liquid interfaces was also investigated. It was found that for highly regular surfaces with small lattice spacing, such as the one used in this paper, the uncertainty introduced was small, but might present a problem for rougher or amorphous surfaces.

Systems with more complex wall structures and Coulomb interactions between solid and liquid constituent molecules are considered as a further research topic. This would include solid surfaces containing hydroxyl groups, which are known to form hydrogen bonds with water molecules, ${ }^{32}$ as well as ion solutes inside the droplet which could potentially form an electric double layer at the interfaces, where the methods developed in this paper should prove particularly effective.

\section{ACKNOWLEDGMENTS}

D.S. is supported by JSPS KAKENHI Grant No. 25807, and Y.Y. is supported by the Ministry of Education, Science, Sports and Culture, Japan, Grant-in-Aid for Young Scientists (B), 22760131, 2010, and Grant-in-Aid for Scientific Research (C), 25420123, 2013. We are also thankful for fruitful discussion with Mr. Satoshi Nakaoka in our group.

\section{APPENDIX: LOCAL ONE-DIMENSIONAL PRESSURE CONTROL}

The method using stochastic velocity rescaling proposed by Bussi et al. ${ }^{25}$ was modified to provide one-dimensional pressure control inside a local region while still preserving the conservation of effective enthalpy $H$. The basic concept is shown in Fig. 15 and the equation of motion is modified in the following way

$$
\begin{gathered}
\dot{r}_{\alpha}^{i}=\frac{\pi_{\alpha}^{i}}{m^{i}}+\eta\left\{r_{\alpha}^{i}-\operatorname{sgn}\left(r_{\alpha}^{i}\right) \cdot l_{\alpha}^{\mathrm{ctrl}}\right\}, \\
\dot{\pi}_{\alpha}^{i}=f_{\alpha}^{i}-\eta \pi_{\alpha}^{i}, \\
\dot{\eta}=\frac{V^{\mathrm{ctrl}}\left(p_{\alpha}^{\mathrm{ctrl}}-p^{\mathrm{set}}\right)+2 k_{B} T^{\mathrm{set}}}{W}, \\
\dot{V}^{\mathrm{ctrl}}=V^{\mathrm{ctrl}} \eta,
\end{gathered}
$$

where $r_{\alpha}^{i}, \pi_{\alpha}^{i}$, and $f_{\alpha}^{i}$ are the $\alpha$ component of coordinates, momentum, and force of particle $i$, while $m^{i}$ is its mass. The variable $\eta$ is proportional to the relative change rate of the control region volume $V^{\mathrm{ctrl}}$. A sign function is used together with the starting position $l_{\alpha}^{\text {ctrl }}$ of the pressure control region to account for the system symmetry. Equations (A1) and (A2) are only 


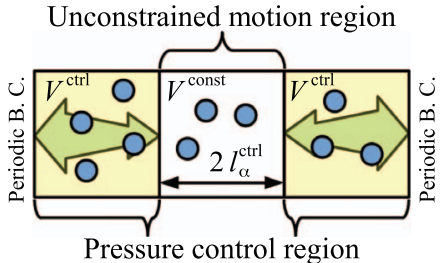

FIG. 15. The concept of a system with one-dimensional pressure control confined to a local region. Particles in the middle white region follow the classical Newtonian equation of motion, while particles in the yellow side region move according to the modified equation of motion. The horizontal dimension of the white region is $2 l_{\alpha}^{\text {ctrl }}$ and its volume is constant, while the horizontal dimension of the yellow region, and thus its volume $V^{\text {ctrl }}$, vary according to its equation of motion. Periodic boundary conditions are set in all lateral directions.

applied in the pressure control direction of particles inside the control region, and classical Newtonian equation of motion is used otherwise. The pressure control region is treated as a single slab and the $\alpha$ component of the local pressure $p_{\alpha}^{\text {ctrl }}$ is calculated there, ${ }^{28}$ while $p^{\text {set }}$ is the system control pressure. The definition of barostat mass $W$ is the same as in the original paper ${ }^{25}$ and $k_{B}$ and $T^{\text {set }}$ are the Boltzmann constant and system control temperature. Temperature is controlled using stochastic velocity rescaling only on particles inside the pressure control region. This provides us with a system where there is only direct pressure control on the outermost regions, while the effective enthalpy $H$ defined in the original paper is still conserved in its unmodified form, and enables us to directly control the pressure of only the liquid phase in systems shown in Fig. 5.

${ }^{1}$ M. K. Chaudhury and G. M. Whitesides, Science 256, 1539 (1992). ${ }^{2}$ S. Daniel, M. K. Chaudhury, and J. C. Chen, Science 291, 633 (2001).

${ }^{3}$ C. Quilliet and B. Berge, Curr. Opin. Colloid Interface Sci. 6, 34 (2001).

${ }^{4}$ Y. T. Cheng, D. E. Rodak, C. A. Wong, and C. A. Hayden, Nanotechnology 17, 1359 (2006).
${ }^{5}$ S. Maruyama, T. Kuruashige, S. Matsumoto, Y. Yamaguchi, and T. Kimura, Microscale Thermophys. Eng. 2, 49 (1998).

${ }^{6}$ J. Z. Tang and J. G. Harris, J. Chem. Phys. 103, 8201 (1995).

${ }^{7}$ F. Bresme and N. Quirke, J. Chem. Phys. 110, 3536 (1999).

${ }^{8}$ P. van Remoortere, J. E. Mertz, L. E. Scriven, and H. T. Davis, J. Chem. Phys. 110, 2621 (1999).

${ }^{9}$ D. Surblys, Y. Yamaguchi, K. Kuroda, T. Nakajima, and H. Fujimura, J. Chem. Phys. 135, 014703 (2011).

${ }^{10}$ M. Tarek and M. L. Klein, J. Phys. Chem. A 101, 8639 (1997).

${ }^{11}$ M. Lundgren, N. L. Allan, and T. Cosgrove, Langmuir 18, 10462 (2002).

${ }^{12}$ T. M. Chang and L. X. Dang, J. Phys. Chem. B 109, 5759 (2005).

${ }^{13}$ N. Nishio, K. Yamana, Y. Yamaguchi, T. Inaba, K. Kuroda, T. Nakajima, K. Ohno, and F. Fujimura, Int. J. Numer. Meth. Fluids 63, 1435 (2010).

${ }^{14}$ H. J. C. Berendsen, J. P. M. Postma, W. F. van Gunsteren, A. DiNola, and J. R. Haak, J. Chem. Phys. 81, 3684 (1984).

${ }^{15}$ W. L. Jorgensen, J. D. Madura, and C. J. Swenson, J. Am. Chem. Soc. 106, 6638 (1984).

${ }^{16}$ W. L. Jorgensen, J. Phys. Chem. 90, 1276 (1986).

${ }^{17}$ L. Fritz and D. Hofmann, Polymer 38, 1035 (1997).

${ }^{18} \mathrm{C}$. Zhang and X. Yang, Fluid Phase Equilib. 231, 1 (2005).

${ }^{19}$ K. Kholmurodov, E. Dushanov, K. Yasuoka, H. Khalil, A. Galal, S. Ahmed, N. Sweilam, and H. Moharram, Natural Sci. 3, 1011 (2011).

${ }^{20}$ H. Yaguchi, T. Yano, and S. Fujikawa, J. Fluid Sci. Technol. 5, 180 (2010).

${ }^{21}$ J. Blömer and A. E. Beylich, Surf. Sci. 423, 127 (1999).

${ }^{22}$ I. P. Omelyan, Comput. Phys. 12, 97 (1998).

${ }^{23}$ N. S. Martys and R. D. Mountain, Phys. Rev. E 59, 3733 (1999).

${ }^{24}$ G. Bussi, D. Donadio, and M. Parrinello, J. Chem. Phys. 126, 014101 (2007).

${ }^{25}$ G. Bussi, T. Zykova-Timan, and M. Parrinello, J. Chem. Phys. 130, 074101 (2009).

${ }^{26}$ M. J. P. Nijmeijer and J. M. J. van Leeuwen, J. Phys. A: Math. Gen. 23, 4211 (1990).

${ }^{27}$ M. J. P. Nijmeijer, C. Bruin, A. F. Bakker, and J. M. J. van Leeuwen, Phys. Rev. A 42, 6052 (1990).

${ }^{28}$ J. G. Weng, S. Park, J. R. Lukes, and C. L. Tien, J. Chem. Phys. 113, 5917 (2000).

${ }^{29}$ T. Young, Trans. R. Soc. Lond. 95, 65 (1805).

${ }^{30}$ J. R. Henderson, P. Tarazona, F. van Swol, and E. Velasco, J. Chem. Phys. 96, 4633 (1992).

${ }^{31}$ F. Leroy, D. J. V. A. dos Santos, and F. Müller-Plathe, Macromol. Rapid Commun. 30, 864 (2009).

${ }^{32}$ K. Yamashita and H. Daiguji, J. Phys. Chem. C 117, 2084 (2013).

${ }^{33}$ G. Vazquez, E. Alvarez, and J. M. Navaza, J. Chem. Eng. Data 40, 611 (1995). 